2005-05

Quantifying drug-induced dyskinesias in the arms using digitised spiral-drawing tasks

\author{
Liu, $X$
}

http://hdl.handle.net/10026.1/3492

10.1016/j.jneumeth.2004.10.005

Journal of Neuroscience Methods

Elsevier BV

All content in PEARL is protected by copyright law. Author manuscripts are made available in accordance with publisher policies. Please cite only the published version using the details provided on the item record or document. In the absence of an open licence (e.g. Creative Commons), permissions for further reuse of content should be sought from the publisher or author. 


\title{
Quantifying drug-induced dyskinesias in the arms using digitised spiral-drawing tasks
}

\author{
Xuguang Liu ${ }^{\mathrm{a}, *}$, Camille B. Carroll ${ }^{\mathrm{b}}$, Shou-Yan Wang ${ }^{\mathrm{c}}$, John Zajicek ${ }^{\mathrm{b}}$, Peter G. Bain ${ }^{\mathrm{a}}$ \\ ${ }^{a}$ Movement Disorders and Neurostimulation Group, Charing Cross Hospital, Division of Neurosciences and Psychological Medicine, \\ 12 East, Fulham Palace Road, Imperial College, London W6 8RF, UK \\ ${ }^{\mathrm{b}}$ Department of Neurology, Derriford Hospital, Plymouth, UK \\ ${ }^{c}$ University Laboratory of Physiology, Oxford, UK
}

Received 26 July 2004; received in revised form 12 October 2004; accepted 12 October 2004

\begin{abstract}
In this study, we quantify the severity of drug-induced dyskinesias in the arms of Parkinson's disease (PD) patients using digitised spiraldrawing tasks. Two spiral drawings, namely a circular and a square spiral, are designed to, respectively, represent the continuous and discrete arm motions, and the size of the spiral is decided so that both the distal and proximal arm joints are involved. Fifteen PD patients, average disease duration $14.4 \pm 7.4$ years, were assessed 30 min after a levodopa challenge whilst performing circular and square spiral-drawing tasks. The velocity of drawing movements was computed and the amplitude of the involuntary dyskinetic movements was measured as the standard deviation of the drawing velocity (SD-DV). The mean amplitude of dyskinetic movements was compared between arms and tasks and was correlated with clinical measures including the Bain dyskinesia scale and the total unified Parkinson's disease rating scale (UPDRS) score. The results showed that there was no statistically significant difference in the amplitude of dyskinesias either between the arms or between the continuous (circular) and discrete (square) spiral drawings in this group of PD patients, but interestingly the interaction between arm and drawing pattern was significant. Significant correlations were found between the magnitude of dyskinesia measured from the spiral-drawing tasks and both the 'on' or 'off' UPDRS and also the Bain dyskinesia scale. We conclude that the drawing tasks may be used to provide an objective method of quantifying the severity of drug-induced dyskinesias in the arm in PD patients.
\end{abstract}

(C) 2004 Elsevier B.V. All rights reserved.

Keywords: Dyskinesia; Drawing; Parkinson's disease; Arm

\section{Introduction}

Drug-induced dyskinesias (Hallett, 2000; Nutt, 2001) in Parkinson's disease (PD) can be very disabling. Dyskinesias in the arms usually appear as irregular involuntary movements involving the shoulder, elbow and wrist, and can be intermingled with other involuntary movements such as tremor. The effective characterisation and quantification of dyskinesia not only improves our understanding of its pathophysiological mechanisms, but also helps diagnosis and the evaluation of treatments. Several quantitative instrumental

\footnotetext{
* Corresponding author. Tel.: +44 2088 467631; fax: +44 2083830663.

E-mail address: x.liu@ic.ac.uk (X. Liu).
}

techniques have been used to quantify dyskinesia including accelerometers, electromyography, force gauges, position transducers, and Doppler ultrasound (for a review, see Hoff et al., 1999). Depending on whether the purpose was clinical or research both ambulatory multi-axial accelerometer (Hoff et al., 2001; Manson et al., 2000) and the laboratory-based movement monitor (Burkhard et al., 1999) techniques have been deployed.

We have previously attempted to quantify involuntary arm movements in PD using visually guided wrist-tracking tasks originally developed to evaluate the efficacy of surgical intervention on action tremor associated with multiple sclerosis (Liu et al., 1997). We found that, following levodopa administration, the drug-induced dyskinesias (mainly in the frequency range of $1-5 \mathrm{~Hz}$ ) showed an increase in ampli- 
tude whereas the action tremor (frequency range of $6-8 \mathrm{~Hz}$ ) showed a decrease (Tubbesing et al., 1999). Furthermore, even though these two types of involuntary arm movement were intermingled during tracking, we were able to selectively study the drug-induced dyskinesias (Liu et al., 2001) and action tremor (Liu et al., 1999) of the wrist in PD patients by testing during a selected medication condition and differentially extracting the component using a proper filter around 5 or $6 \mathrm{~Hz}$ on the compound tracking signal.

Various drawing tasks have been studied (Fukushi and Ashe, 2003; Lacquaniti, 1989; Mergl et al., 1999; Moran and Schwartz, 1999; Reina and Schwartz, 2003) and clinically, scoring the patient's drawing performance is a convenient and effective way to assess the severity of motor dysfunctions such as tremor of the upper limb (Bain et al., 1993). Digitising tablets can be used to quantify normal (Lacquaniti et al., 1987; Mergl et al., 1999) and tremulous arm movements (Elble et al., 1996; Erasmus et al., 2001; Pullman, 1998). Spiral drawing may be particularly suited to quantifying involuntary arm movements involving more than just a single joint, as in order to draw a spiral in $2 \mathrm{D}$ both the distal and proximal arm joints must be used. In addition, the degree of involvement of the arm joints alters as the diameter of the drawing movements changes; so that the larger the limb movement, the smaller the magnitude of the contributions obtained by motions at distal relative to proximal joints (Lacquaniti et al., 1987; Meulenbroek et al., 1993). Given that in PD, drug-induced dyskinesias may involve all the joints in the arm the dyskinesias are better represented in a spiral-drawing task rather than our previous tracking task in which the patient's movements were largely restricted to the wrist (Liu et al., 1999, 2001; Tubbesing et al., 1999), because with spirals there are changes in the overall size, direction and velocity of the drawing movements that widely recruit muscle groups and joints in the arm.

Another issue that needs to be considered in using drawing tasks to quantify dyskinetic arm movements for PD patients is the effect of their Parkinsonism on their voluntary drawing movements; as these may in turn influence the assessment of dyskinesias during drawing (Brown et al., 1999; Wenzelburger et al., 2003). In particular, PD patients may have more difficulty performing drawings in which changes of movement direction and velocity are discrete compared to gradual and continuous. To assess the possible interaction between the voluntary movement involved in drawings and the dyskinesias, two patterns, namely a circular and a square spiral, were designed to, respectively, represent the continuous and discrete motions.

In the present study, we assessed the clinical relevance of drawing tasks as a method of quantifying drug-induced dyskinesia by correlating the indices derived from the drawings, representing dyskinesias, with the clinical assessments that were also performed simultaneously. The total unified Parkinson's disease rating scale (UPDRS) score was recorded whilst patients were in the 'off-medication' and then 'onmedication' state. Furthermore, the results obtained with the spiral-drawing tasks were also correlated with a dyskinesiaspecific assessment, the Bain dyskinesia scale.

\section{Method}

\subsection{Subjects and clinical assessments}

Sixteen patients, aged 18-78, with a clinical diagnosis of idiopathic PD and average disease duration $14.4 \pm 7.4$ years, were recruited from PD outpatient clinics in Devon and Cornwall, UK. Inclusion criteria were levodopa-induced dyskinesia rated at $\geq 2$ (out of 12) on questions 32-34 of the UPDRS and fixed anti-Parkinsonian medication for at least 1 month prior to the study, which was primarily a clinical trial examining the effect of cannabis on PD dyskinesia (Carroll et al., 2004). The study received ethical approval from the Plymouth Local Research Ethics Committee, UK, and written informed consent was obtained from all participants prior to enrollment. Six healthy subjects, aged $25-45$, were recruited to serve as controls.

The following clinical assessments were carried out: (i) the total UPDRS score was recorded whilst patients were in the off-medication and then on-medication state and (ii) dyskinesia-specific assessments using the Bain dyskinesia scale, whilst the patients were in the on-medication state.

\subsection{Drawing tasks and signal acquisition}

Templates of a circular and a square spiral were made on a A4-size paper and placed underneath a transparent plastic sheet fixed on the surface of a digital graphic tablet (Easypen G6, Netway Components Limited, Kent, UK) which was connected to a laptop PC. The circular spirals had maximal outside diameter of $100 \mathrm{~mm}$ and the square spiral had a maximum outside length segment of $100 \mathrm{~mm}$. Both spirals involved four cycles each with an incremental change of $14 \mathrm{~mm}$ in diameter between turns (Fig. 1a). Before each recording, the centre point of each spiral was registered first by placing the pen onto the centre of the template. The patients and normal controls were instructed to hold a pen and trace the spiral templates at a self-paced velocity. Each drawing was performed from the centre outwards and then inwards back to the centre. Thus the drawings were performed in both clock- and anti-clockwise directions. The drawings were made without allowing the forearm to rest on the drawing board and both arms were tested alternately. The pen position was continuously recorded and the drawing signal was digitised at a rate of $50 \mathrm{~Hz}$. Drawings were displayed on-line on the PC screen and the data were saved onto a hard disk for analysis off line. For the PD patients the drawings were recorded when an examiner (PGB) considered the dyskinesias to be prominent, which was usually at least $30 \mathrm{~min}$ after the levodopa challenge. The test dose of levodopa used the dispersible formulation (normal early 

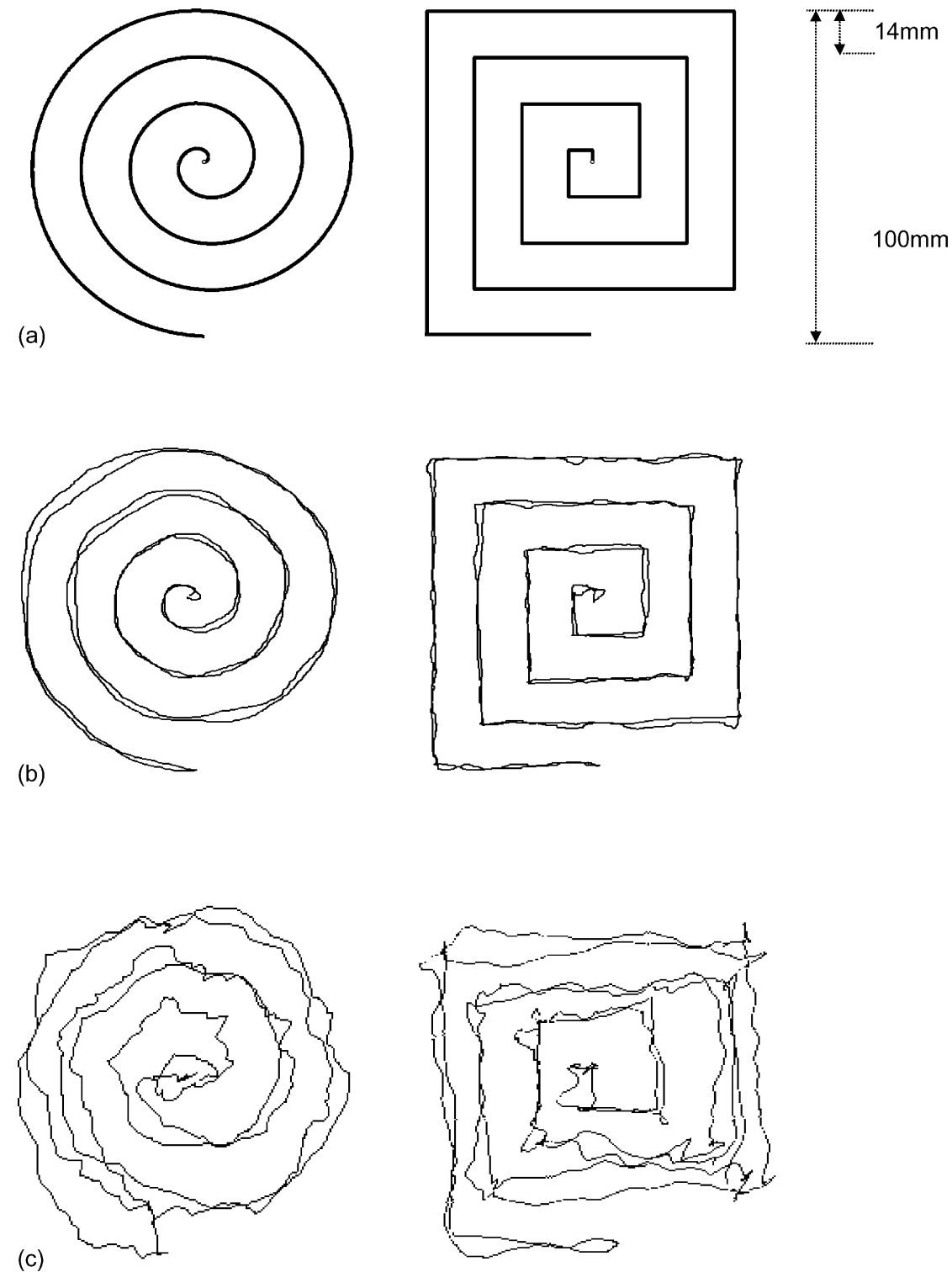

Fig. 1. (a) Templates of circular and square spirals; (b) examples of spiral drawing of a healthy control; and (c) examples of spiral drawing of a patient with levodopa-induced dyskinesias.

morning dose + adjustment for normal dose of dopamine agonist $+25 \%)$.

\subsection{Signal analysis}

The signals obtained from the spiral drawings were filtered with a zero phase, four-pole Butterworth filter with corner frequency $25 \mathrm{~Hz}$. The position signals of the pen in horizontal, vertical, radial and tangential directions were differentiated to yield velocity signals. The power spectrum of the velocity signal in each drawing direction was obtained using fast Fourier transform over segments of $5 \mathrm{~s}$. The velocity signals were then bandwidth filtered in the range of $1-5 \mathrm{~Hz}$ to extract the dyskinetic movement so that contamination by the accompanying voluntary drawing movements $(<1.0 \mathrm{~Hz})$ and action tremor $(>5 \mathrm{~Hz})$ could be eliminated. Then the standard deviation of drawing velocity (SD-DV) for each direction was computed from the extracted velocity signals representing dyskinesias. All signals were processed using MATLAB (Version 6.5, MathWorks, Inc., Natick, MA, USA).

\subsection{Statistical analysis}

The mean SD-DV value averaged across two directions was used to represent each of the two drawing patterns. The difference in the SD-DV between the drawing patterns and hand sides were tested using analysis of variance (ANOVA) across 16 patients with a completely-within design as all of the measurements were obtained from the same patient group. Correlations between the SD-DV and clinical assessments 


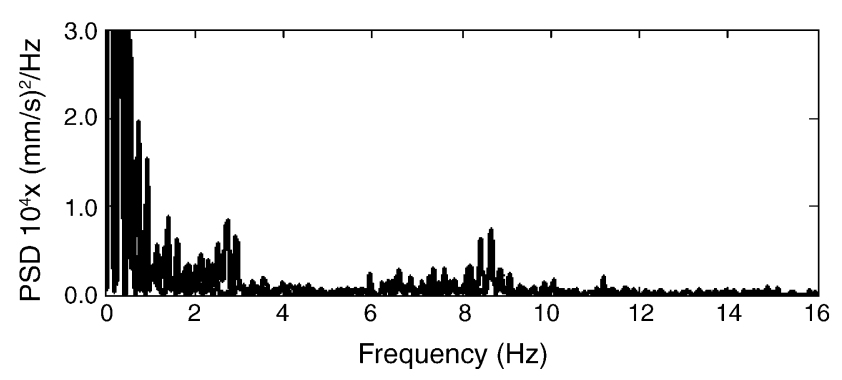

Fig. 2. A power spectrum of a patient's spiral drawing. Voluntary drawing movements $(<1.0 \mathrm{~Hz})$, drug-induced dyskinesias $(1-5 \mathrm{~Hz})$ and action tremor $(6-10 \mathrm{~Hz})$ can then be differentially extracted.

were analysed using linear regression with a confidence range of $95 \%$ and its statistical significance was tested on the correlation coefficients.

\section{Results}

The drawings of normal controls (Fig. 1b) were smoother with much less error than the drawings of the patients (Fig. 1c), which were characterised by irregular errors around the defined trajectories of the drawing templates (Fig. 1a). Power spectra of the drawing velocity data in the normal controls showed the voluntary drawing movements were predominantly located in the frequency range of $<1.0 \mathrm{~Hz}$. Among the PD patients, drawings showed three distinct components in the frequency spectrum: (1) similar to normal drawings, the voluntary drawing movements with a frequency range $<1.0 \mathrm{~Hz}$; (2) irregular dyskinetic movements with a broad peak ranging from 1 to $5 \mathrm{~Hz}$; and (3) action tremor oscillating around $6-10 \mathrm{~Hz}$ and usually having a sharp peak (Fig. 2). A filter with a bandwidth of $1-5 \mathrm{~Hz}$ was then added before computing the SD-DV in order to subtract the dyskinetic movements and reduce contamination by the co-existing voluntary drawing movements and action tremor.

The averaged SD-DV values of the circular and square spiral drawings made by the left $(11.75 \pm 5.85 \mathrm{~mm} / \mathrm{s}$, circular; $9.52 \pm 4.04 \mathrm{~mm} / \mathrm{s}$, square) and right $(9.89 \pm 4.30 \mathrm{~mm} / \mathrm{s}$, circular; $11.00 \pm 6.37 \mathrm{~mm} / \mathrm{s}$, square) arms of the 16 PD patients were statistically tested using two-factor ANOVA. No significant difference was found between: (i) the left and right arms $(P<0.47)$; (ii) the circular and square drawings $(P<0.84)$; but (iii) a significant difference was found in the interaction between arm and drawing pattern $(P<0.02)$. This result indicates that the left arm has higher SD-DV scores for circular drawings than the right arm, whereas the right arm has higher SD-DV scores for square drawing than the left arm. The hand difference appears to be most obvious for the circular drawings specifically.

Significant correlations were found between the SD-DV and the scores obtained from the on-medication UPDRS $(r=0.63, P<0.02, n=16$; Fig. 3A) and off-medication UPDRS ( $r=0.64, P<0.02, n=16$; Fig. 3B), and the Bain dyskinesia scale $(r=0.65, P<0.01, n=16$, Fig. $3 \mathrm{C})$.
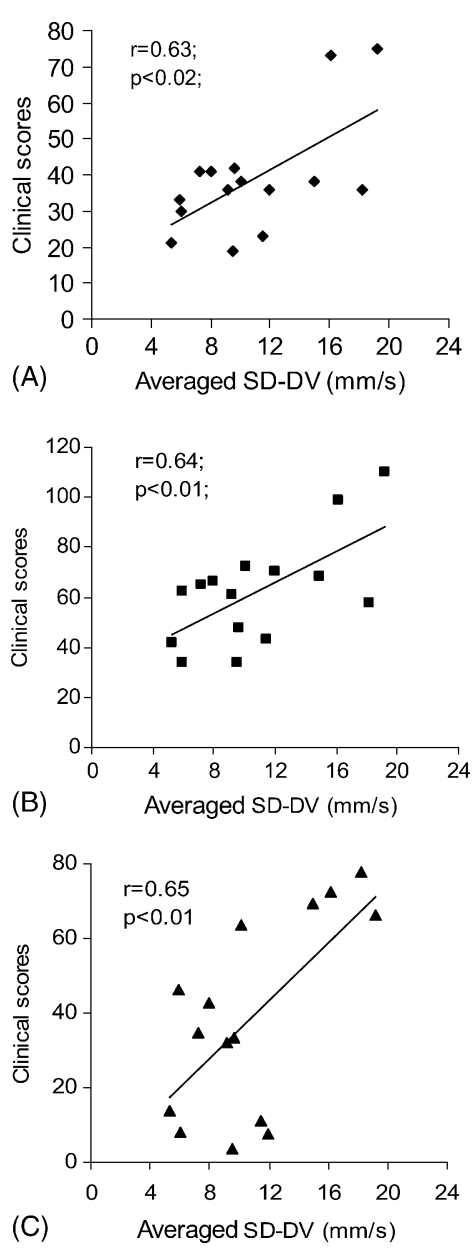

Fig. 3. Significant correlations of the mean standard deviation of movement velocity (SD-DV) of the drawings with the UPDRS medication-on (A), medication-off (B) and Bain dyskinesia (C) scores.

\section{Discussion}

In this study, we quantified drug-induced dyskinesias in the arms of PD patients whilst they were drawing circular and square spirals in 2D using a digital tablet. The following aspects of upper limb Parkinsonian dyskinesias were considered in the design of this method. First, the spiral-drawing tasks involved changes in drawing diameter and direction that allow deficits in the movement and coordination of all the joints in the arm to be detected and the movement in $2 \mathrm{D}$ (for instance, radial/tangential in circular or horizontal/vertical in square spiral drawings) to be analysed. Second, the position of the drawing pen was continuously recorded and digitised at a fixed rate, allowing temporal in addition to spatial information of the arm movement to be obtained and analysed in the time and frequency domains. In addition, the method requires very limited hardware which is readily transportable, so that patients could even be assessed in their own homes.

We designed two patterns of spiral, namely the circular and square spirals, equivalent in overall size, number of turns and increment distance. By comparing drawings performed 
on these two patterns, we examined the possible difference in dyskinesia amplitude that may be induced by the pattern of voluntary drawing movements. As a result of bradykinesia, PD patients might have displayed more deficits when drawing a spiral in which changes in movement direction and velocity are discrete compared to when a gradual and continuous motion is required. However, our results showed that no significant difference was found in the SD-DV score between these two patterns of spiral drawing in this group of PD patients. This may imply that bradykinesia in the patients that we studied were largely overcome by dopaminergic medication, as patients were tested for dyskinesias during their on-medication state. On the other hand, one factor that might have compromised detection of a differential bradykinetic effect was that visual guidance was provided throughout the test as the patients were essentially tracing a visually presented template. Bradykinesia, a consequence of dysfunction of the basal ganglia, is more profound when no visual cues are available as the involvement of the cerebellar pathways are then largely redundant (Jueptner and Weiller, 1998). Furthermore in our drawing tasks the overall amplitude of dyskinesias may have been overestimated because of the presence of visually dependent voluntary error correcting movements (around $1.7-2 \mathrm{~Hz}$ ). These are a common feature associated with on-line visual feedback control of normal slow manual movements in healthy subjects (Miall et al., 1993), and in patients with cerebellar dysfunction (Liu et al., 1997) and drug-induced dyskinesias (Liu et al., 2001). This possible source of error was not excluded from the signal (frequency range from 1 to $5 \mathrm{~Hz}$ ) even though the voluntary drawing movements $(<1 \mathrm{~Hz})$ were digitally filtered out.

Interestingly, the interaction of arm and drawing pattern in the SD-DV score was found to be statistically significant although there were no significant differences between arms and drawing patterns, respectively. This indicates that the left arm has higher SD-DV scores for circular drawings than the right arm, whereas the right arm has higher SD-DV scores for square drawing compared to the left arm. The difference between arms was particularly obvious for the circular drawings. This interaction measurement may be a more sensitive measure of dyskinesias because it may accommodate factors such as asymmetry of Parkinsonian dyskinesia between arms and the different controlling mechanisms for drawing different patterns.

The most important issue addressed by this study was assessing the validity and clinical relevance of quantifying upper limb drug-induced dyskinesias using the spiral-drawing tasks. To examine this, we took the approach of correlating the overall drawing results with various clinical assessments. To represent the overall dyskinesias for each patient's drawings, we calculated the mean SD-DV by averaging over radial and tangential directions for circular drawing and horizontal and vertical directions for the square drawings for each arm, and then the mean SD-DV by averaging data from both arms. Although we reduced the detail in our analysis of the spiral drawings, the data based on the mean value from the draw- ing task results obtained in each patient showed significant correlation not only to the overall UPDRS scores both onand off-medication, representing Parkinsonism in general, but also the scores on the Bain dyskinesia scale which specifically scores dyskinesias. On the other hand, depending on the specific purpose of the assessment, a more detailed analysis would be appropriate, e.g. to study the interaction between voluntary drawing and involuntary dyskinetic movements.

The methods described in this paper were applied to the assessment and quantification of upper limb drug-induced dyskinesia in a randomised double-blind clinical trial of cannabis for dyskinesia in PD (Carroll et al., 2004). In addition to all the available patient- or physician-based clinical assessments for dyskinesias, the method described here provided specific quantitative information about dyskinesias in the arms whilst drawing. These drawing tasks can potentially be used to quantify other involuntary movements that involve the whole arm such as intention tremor or chorea and may assist in their diagnosis as well as the evaluation of medical or surgical treatments.

\section{Acknowledgements}

We would like to express our gratitude to L. Teare, C. Joint, C. Wroath, S. Parkin, P. Fox and J. Hobart for their help in patient care, and to the patients for their help with this study.

\section{References}

Bain PG, Mally J, Gresty M, Findley LJ. Assessing the impact of essential tremor on upper limb function. J Neurol 1993;241:54-61.

Brown RG, Dowsey PL, Brown P, Jahanshahi M, Pollak P, Benabid AL, et al. Impact of deep brain stimulation on upper limb akinesia in Parkinson's disease. Ann Neurol 1999;45:473-88.

Burkhard PR, Shale H, Langston JW, Tetrud JW. Quantification of dyskinesia in Parkinson's disease: validation of a novel instrumental method. Mov Disord 1999;14:754-63.

Carroll CB, Bain PG, Teare L, Liu X, Joint C, Wroath C, Parkin S, Fox P, Wright D, Hobart J, Zajicek J. Cannabis for dyskinesia in Parkinson's disease: a randomised double-blind crossover study. Neurology 2004;63:1245-50.

Elble RJ, Brilliant M, Leffler K, Higgins C. Quantification of essential tremor in writing and drawing. Mov Disord 1996;11:70-8.

Erasmus LP, Sarno S, Albrecht H, Schwecht M, Pollmann W, Konig N. Measurement of ataxic symptoms with a graphic tablet: standard values in controls and validity in multiple sclerosis patients. J Neurosci Meth 2001;108:25-37.

Fukushi T, Ashe J. Adaptation of arm trajectory during continuous drawing movements in different dynamic environments. Exp Brain Res 2003;148:95-104.

Hallett M. Clinical physiology of dopa dyskinesia. Ann Neurol 2000;47:147-50

Hoff JI, van den Plas AA, Wagemans EA, van Hilten JJ. Accelerometric assessment of levodopa-induced dyskinesias in Parkinson's disease. Mov Disord 2001;16:58-61.

Hoff JI, van Hilten BJ, Roos RA. A review of the assessment of dyskinesias. Mov Disord 1999;14:737-43. 
Jueptner M, Weiller C. A review of differences between basal ganglia and cerebellar control of movements as revealed by functional imaging studies. Brain 1998;121:1437-49.

Lacquaniti F. Central representations of human limb movement as revealed by studies of drawing and handwriting. Trends Neurosci 1989;12:287-91.

Lacquaniti F, Ferrigno G, Pedotti A, Soechting JF, Terzuolo C. Changes in spatial scale in drawing and handwriting: kinematic contributions by proximal and distal joints. J Neurosci 1987;7:819-28.

Liu X, Miall C, Aziz TZ, Palace JA, Haggard PN, Stein JF. Analysis of action tremor and impaired control of movement velocity in multiple sclerosis during visually guided wrist-tracking tasks. Mov Disord 1997;12:992-9.

Liu X, Osterbauer R, Aziz TZ, Miall RC, Stein JF. Increased response to visual feedback of drug-induced dyskinetic movements in advanced Parkinson's disease. Neurosci Lett 2001;304:25-8.

Liu X, Tubbesing SA, Aziz TZ, Miall RC, Stein JF. Effects of visual feedback on manual tracking and action tremor in Parkinson's disease. Exp Brain Res 1999;129:477-81.

Manson AJ, Brown P, O'Sullivan JD, Asselman P, Buckwell D, Lees AJ. An ambulatory dyskinesia monitor. J Neurol Neurosurg Psychiatr 2000;68:196-201.

Mergl R, Tigges P, Schroter A, Moller HJ, Hegerl U. Digitized analysis of handwriting and drawing movements in healthy subjects: methods, results and perspectives. J Neurosci Meth 1999;90:15769.

Meulenbroek RG, Rosenbaum DA, Thomassen AJ, Schomaker LR. Limbsegment selection in drawing behaviour. Quart J Exp Psychol: Hum Exp Psychol 1993;46A:273-99.

Miall RC, Weir DJ, Stein JF. Intermittency in human manual tracking tasks. J Motor Behav 1993;25:53-63.

Moran DW, Schwartz AB. Motor cortical activity during drawing movements: population representation during spiral tracing. J Neurophysiol 1999;82:2693-704.

Nutt JG. Motor fluctuations and dyskinesia in Parkinson's disease. Parkinsonism Relat Disord 2001;8:101-8.

Pullman SL. Spiral analysis: a new technique for measuring tremor with a digitizing tablet. Mov Disord 1998;13(Suppl 3):85-9.

Reina GA, Schwartz AB. Eye-hand coupling during closed-loop drawing: evidence of shared motor planning? Hum Mov Sci 2003;22:137-52.

Tubbesing SA, O’Neill S, Liu X, Miall RC, Aziz TZ, Stein JF. Application of visually guided wrist and arm tracking tasks in study of involuntary movements in Parkinson's disease (PD). J Physiol (London) 1999;515P:153P.

Wenzelburger R, Kopper F, Zhang BR, Witt K, Hamel W, Weinert D, et al. Subthalamic nucleus stimulation for Parkinson's disease preferentially improves akinesia of proximal arm movements compared to finger movements. Mov Disord 2003;18:1162-9. 